\title{
Degeneracy and Strong Fluctuation-Induced First-Order Phase Transition in the Dipolar Pyrochlore Antiferromagnet
}

\author{
O. Cépas ${ }^{1}$, A. P. Young ${ }^{2}$ and B. S. Shastry ${ }^{2}$ \\ 1 Institut Laue Langevin, BP 156, F-38042 Grenoble Cedex 9, France. \\ 2 Department of Physics, University of California, Santa Cruz, 95064.
}

(Dated: October 30, 2018)

\begin{abstract}
We show that a continuous set of degenerate critical soft modes strongly enhances the firstorder character of a fluctuation-induced first-order transition in the pyrochlore dipolar Heisenberg antiferromagnet. Such a degeneracy seems essential to explain the strong first-order transition recently observed in $\mathrm{Gd}_{2} \mathrm{Sn}_{2} \mathrm{O}_{7}$. We present some evidence from Monte-Carlo simulations and a perturbative renormalization group expansion.
\end{abstract}

\section{INTRODUCTION}

In this paper we study phase transitions in Heisenberg magnets on the pyrochlore lattice, which consists of corner sharing tetrahedra ${ }^{1,2}$. The motivation is partly theoretical, to understand the behavior of highly frustrated systems when there is a degeneracy, or near degeneracy, between different ordered states. There is also experimental motivation since experiments on $\mathrm{Gd}_{2} \mathrm{Sn}_{2} \mathrm{O}_{7}$ and $\mathrm{Gd}_{2} \mathrm{Ti}_{2} \mathrm{O}_{7}$ have shown a rich behavior, ${ }^{3,4}$ including multiple field transitions ${ }^{5,6}$ which we would like to understand.

For the family of rare earth pyrochlore systems it is well known ${ }^{3}$ that dipole-dipole interactions are important, since the angular momentum is large $(S=7 / 2$ for $\mathrm{Gd}$ ). If one adds nearest neighbor exchange to dipoledipole interactions, the Fourier transform of the total interaction $J(\mathbf{q})$ is virtually independent ${ }^{7}$ of $\mathbf{q}$ (and takes its minimum value) along the $(1,1,1)$ directions of the reciprocal space. $^{3,8}$ This means that the magnetic ordering wave-vector could, potentially lie anywhere along these lines.

It turns out that the phases at the endpoints, $\mathbf{q}=\mathbf{0}$ (denoted, following Ref. 10 , by $\mathrm{A}$ ) and $\mathbf{q}=(\pi, \pi, \pi)$ (denoted by $\boldsymbol{\pi}$ or B) are particularly important. The ordering expected at $\mathbf{q}=\mathbf{0}$ (A-type) has been discussed by several authors ${ }^{3,5,8,10}$ and is shown in Fig. 1. A possible ordering at $\mathbf{q}=\boldsymbol{\pi}$ (B-type) has been proposed in Ref. [10].

Although one would imagine that $\mathrm{Gd}_{2} \mathrm{Sn}_{2} \mathrm{O}_{7}$ and $\mathrm{Gd}_{2} \mathrm{Ti}_{2} \mathrm{O}_{7}$ should be quite similar, since the crystal structures are the same (apart from a very small difference in the lattice constant), it is found that $\mathrm{Gd}_{2} \mathrm{Sn}_{2} \mathrm{O}_{7}$ has a strong first-order transition, ${ }^{4}$ while $\mathrm{Gd}_{2} \mathrm{Ti}_{2} \mathrm{O}_{7}$ has a second-order transition. ${ }^{12}$ Furthermore, $\mathrm{Gd}_{2} \mathrm{Ti}_{2} \mathrm{O}_{7}$ orders at $\mathbf{q}=\boldsymbol{\pi}^{12,13}$ while $\mathrm{Gd}_{2} \mathrm{Sn}_{2} \mathrm{O}_{7}$ appears more compatible with the A phase. ${ }^{14}$ While the small change in lattice parameter between $\mathrm{Gd}_{2} \mathrm{Sn}_{2} \mathrm{O}_{7}$ and $\mathrm{Gd}_{2} \mathrm{Ti}_{2} \mathrm{O}_{7}$ could change somewhat the exchange constants, it seems remarkable that the nature of the ordering changes so dramatically. We would like to understand such a delicate dependence of ordering on exchange constants.

The first order nature of the transition observed in $\mathrm{Gd}_{2} \mathrm{Sn}_{2} \mathrm{O}_{7}$ is at variance with mean-field theory which predicts $^{10}$ a second-order transition. In order to clarify

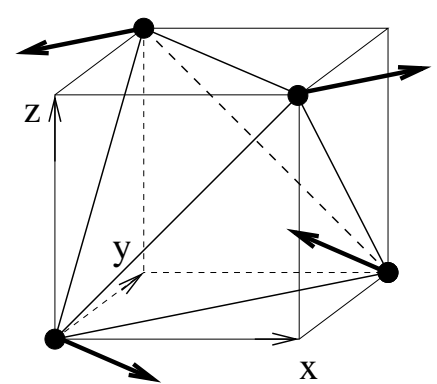

FIG. 1: The A state $(\mathbf{q}=\mathbf{0})$ of the pyrochlore lattice stabilized at low temperatures by the dipole-dipole interactions (Refs. $[3,5,8,10]$ ). Since the ordering is at $\mathbf{q}=0$, all tetrahedra have the same spin configuration as the one shown. In the figure, all the spins lie onto the $(x y)$ plane and form pairs of antiparallel spins that are parallel to the opposite edge of the tetrahedron they belong to. There are equivalent $(x z)$ and $(y z)$ states. The magnetic order is therefore characterized by a $n=3$-component order parameter, $\psi$ with $(x y)$ corresponding to $\psi=(1,0,0)$ at $\mathrm{T}=0$.

the order of the transition, and to see whether it is affected by longer range exchange interactions which lift the degeneracy of $J(\mathbf{q})$, we have performed Monte Carlo simulations of the classical dipolar Heisenberg model on the pyrochlore lattice. These show that the apparent order of the transition is indeed very sensitive to the exchange constants.

We have supplemented the numerics by a perturbative renormalization group $(\mathrm{RG})$ analysis. It is known that "fluctuation-induced first-order transitions" occur when there is no stable fixed point in a perturbative RG calculation. This frequently occurs when the number of components of the order parameter $n$ is larger than $4,{ }^{15-18}$. In general, such transitions are expected to be only weakly first-order. Another microscopic scenario for a first order transition is the proposal of Brazovskii ${ }^{19}$ that the existence of a continuous set of degenerate soft modes could change the order of the transition. It was latter shown that the RG analysis of models with soft modes along special directions lacks stable fixed points and the models are indeed likely to undergo first-order transitions. ${ }^{20,21}$ This scenario is, for instance, relevant to the description of the liquid crystal transition where the degeneracy naturally comes from the isotropy of the liquid. ${ }^{20}$ It is also 
particularly relevant in frustrated magnets where frustration may, precisely, provide a large number of quasidegenerate soft-modes, though with different geometrical structures. We shall consider a similar RG analysis, applicable for the symmetry of the dipolar pyrochlores which have lines of degeneracy, in this paper.

In this work, we study the order of the transition of the classical Heisenberg model on the pyrochlore lattice with long-range dipole-dipole interactions and exchange interactions. We show, by means of Monte-Carlo simulations, that the transition is strongly first-order when a continuous set of soft modes is present in $J(\mathbf{q})$, and becomes weakly first-order when that degeneracy is removed by including further neighbor interactions (Sec. II). In section III, we present a Landau-Ginzburg-Wilson simplified model and a perturbative renormalization group analysis of the transition, that predicts a first-order transition, in agreement with the numerical results in Sec. II. We summarize our conclusions in Sec. IV.

\section{MONTE-CARLO SIMULATIONS}

We present in this section some results of Monte Carlo simulations, using the parallel tempering approach, ${ }^{22}$ on the Heisenberg model with dipolar and exchange interactions on the pyrochlore lattice.

The classical Heisenberg Hamiltonian is:

$$
\begin{aligned}
\mathcal{H} & =\sum_{\langle i, j\rangle} J_{i j} \mathbf{S}_{i} \cdot \mathbf{S}_{j} \\
& +\left(g \mu_{B}\right)^{2} \sum_{\langle i, j\rangle}\left(\frac{\mathbf{S}_{i} \cdot \mathbf{S}_{j}}{r_{i j}^{3}}-3 \frac{\left(\mathbf{r}_{i j} \cdot \mathbf{S}_{i}\right)\left(\mathbf{r}_{i j} \cdot \mathbf{S}_{j}\right)}{r_{i j}^{5}}\right)
\end{aligned}
$$

where $\mathbf{S}_{i}$ is a classical spin vector of length $S=7 / 2$ (for $\mathrm{Gd}^{3+}$ ) on site $i$, and $J_{i j}$ is the Heisenberg exchange between the neighbors: we will consider the first $(J)$, second $\left(J_{2}\right)$ and third neighbor $\left(J_{3}\right)$ couplings. ${ }^{10}$

The number of spins is $N=16 L^{3}(L \leq 4)$ and periodic boundary conditions are applied. The factor of 16 arises because the pyrochlore lattice consists of an fcc lattice of tetrahedra, each tetrahedron has 4 spins, and there are 4 sites of the fcc lattice in the conventional cubic cell. To incorporate the B-phase with periodic boundary conditions, we need $L$ to be even, so most of our results are for $L=2$ and 4 . Often the long-range dipolar interactions are cut-off beyond a couple of neighbors ${ }^{23}$ to speed up the simulations. However, here we have kept a large number of neighbors (practically infinite) in order to reproduce accurately the structure of the degenerate states. If the dipole-dipole interaction is cut-off, ripples appear in the degenerate lines of soft modes ${ }^{3,10,11}$. We constructed periodic repetitions of the Monte Carlo clusters and included the contributions of many blocks in performing the dipolar sums. Because there is no cut-off in the dipole interactions, the simulation becomes slow for large sizes, so we are limited to $L \leq 4(N \leq 1024)$.
We investigate ordering at $\mathbf{q}=0$ (called A) see Fig. 1, and at $\mathbf{q}=\boldsymbol{\pi}$ (called B), see Ref. [10]. With A ordering the order parameter has $n=3$ components and with $\mathrm{B}$ ordering it has $n=4$ components as detailed in Sec. III. The $n$-component order parameters are given by

$$
\begin{aligned}
\psi & =\left(\psi_{1}, \ldots, \psi_{n}\right) \\
\psi_{\alpha} & =\frac{1}{N} \sum_{i=1}^{N} \mathbf{S}_{i} \cdot \mathbf{e}_{i}^{(\alpha)}
\end{aligned}
$$

where $\mathbf{e}_{i}^{(\alpha)}$ is the unit vector of spin $i$ assuming the system is fully ordered in component $\alpha$ of ordering type A with the $(x y)$ state (resp. $(x z),(y z))$ corresponding to $\psi=(1,0,0)$ (resp. $\psi=(0,1,0), \psi=(0,0,1))$ (see e.g. the arrows in Fig. 1) or B. In the course of the simulation of a finite system, the spin configuration can fluctuate between different, equivalent ordered states. We therefore compute the invariant quantities

$$
m^{(2)}=\sum_{\alpha=1}^{n}\left\langle\psi_{\alpha}^{2}\right\rangle ; \quad m^{(4)}=\left\langle\left(\sum_{\alpha=1}^{n} \psi_{\alpha}^{2}\right)^{2}\right\rangle
$$

It is convenient to also compute the dimensionless Binder ratio

$$
g=\frac{1}{2}\left[(n+2)-n \frac{m^{(4)}}{\left(m^{(2)}\right)^{2}}\right]
$$

for both A and B orderings, which has the property that it tends to 0 at high temperature and to 1 in an ordered state. (Remember that $n=3$ for A-type ordering and $n=4$ for B-type ordering.)

Firstly we consider the dipolar model with only nearest neighbor interactions. Results for the order parameters are shown in Fig. 2. The parameter $J$ is taken to be the same as in $\mathrm{Gd}_{2} \mathrm{Ti}_{2} \mathrm{O}_{7}$, i.e. from the high temperature susceptibility $J=0.4 \mathrm{~K}$, and the dipole-dipole interactions are fixed by the inter-ion distances. ${ }^{3,4}$ The order parameter associated with the A state jumps rapidly at $T \simeq 0.7$ $\mathrm{K}$, indicating a strong first order transition at that temperature, whereas that of the B state goes to zero at the same temperature. These results gives a much lower value of the transition temperature $T_{N}$ than the meanfield theory value of $5.3 \mathrm{~K}$, though it is a bit smaller than the experimental value of $1 \mathrm{~K}$.

The Binder ratio shown in Fig. 3 is also strongly discontinuous. In the vicinity of $T_{c}$ the Binder ratio gets negative, as expected for a first-order phase transition. ${ }^{24}$ Given the results of Figs. 2 and 3, we conclude that the transition is strongly first-order for $J_{2}=J_{3}=0$. The very small preference for the B-phase ${ }^{9-11}$ indicated by the small minimum of $J(\mathbf{q})$ at $\mathbf{q}=\boldsymbol{\pi}$, which would be relevant at a second order transition, is unimportant here because the transition is so strongly first order.

We now include further neighbor interactions $J_{2}$ and $J_{3}$ that lift the degeneracy of the dipolar model and select other states, as studied in detail in Ref. [10]. The lowest 


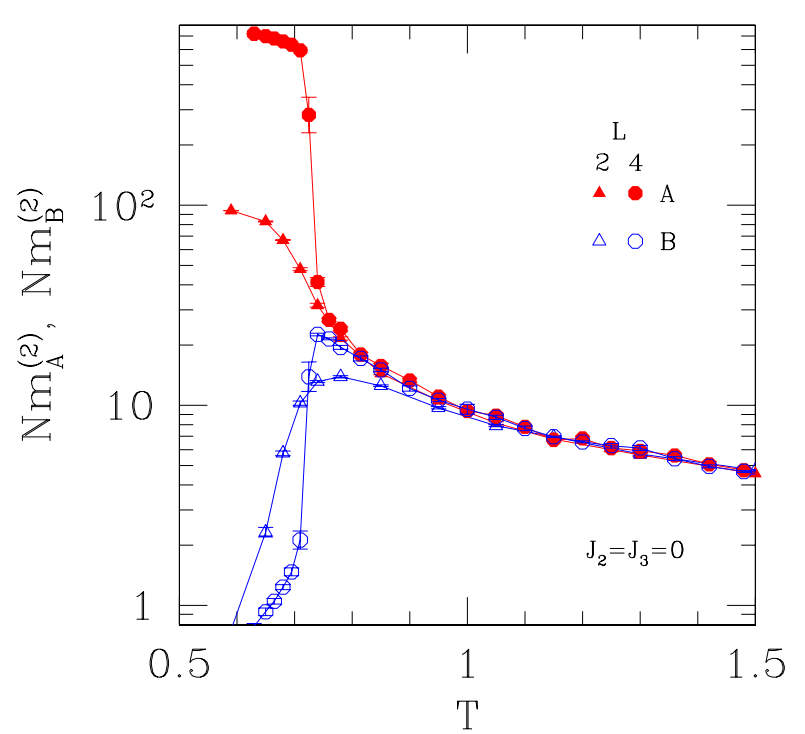

FIG. 2: (Color online). Order parameter squared $(\times N)$ for the A phase, shown in Fig. 1 , and the B phase, for $J_{2}=J_{3}=0$ as function of temperature $T$ in a Monte Carlo simulation of the dipolar pyrochlore Heisenberg antiferromagnet. We see a large jump in the order parameter for the largest system size $L=4$ signaling the onset of a strong first-order transition. The number of spins is given by $N=16 L^{3}$.

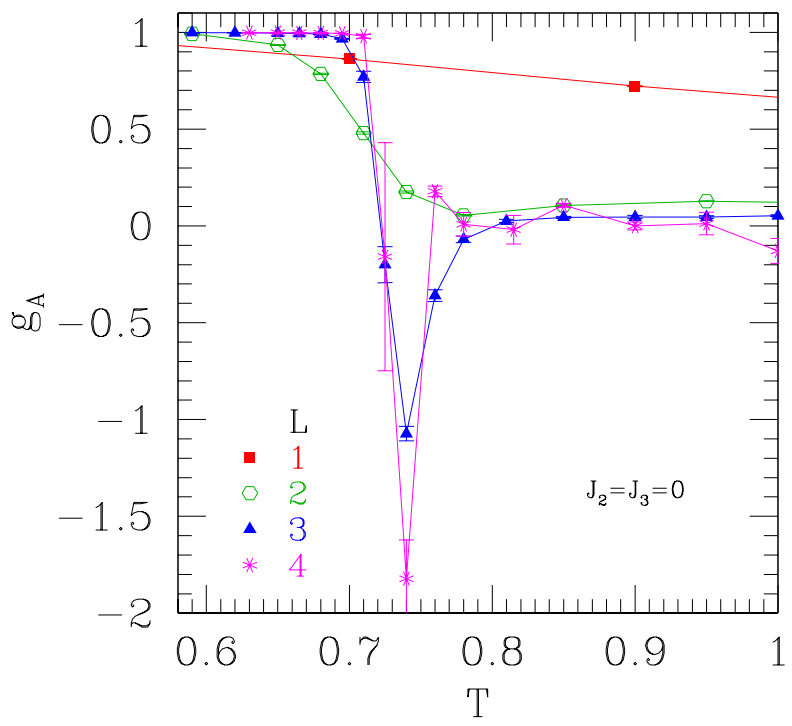

FIG. 3: (Color online). Binder ratio for the A phase for different system sizes $L$ for $J_{2}=J_{3}=0$.

part of the spectrum of $J(\mathbf{q})$ is shown in Fig. 4 for $\mathbf{q}$ along the $(1,1,1)$ direction. We see that spectrum is almost precisely degenerate for $J_{2}=J_{3}=0$ but that $\mathbf{q}=0$ is preferred if $J_{2}$ and $J_{3}$ are negative, while $\mathbf{q}=\boldsymbol{\pi}$ is preferred if $J_{2}$ and $J_{3}$ are positive. For simplicity we restrict ourselves to $J_{2}=J_{3}$ and study how the character of the transition is modified relative to the case $J_{2}=J_{3}=$ 0 .

For $J_{2}=J_{3}<0$, we find the same A state as for $J_{2}=J_{3}=0$, but the transition temperature shifts to

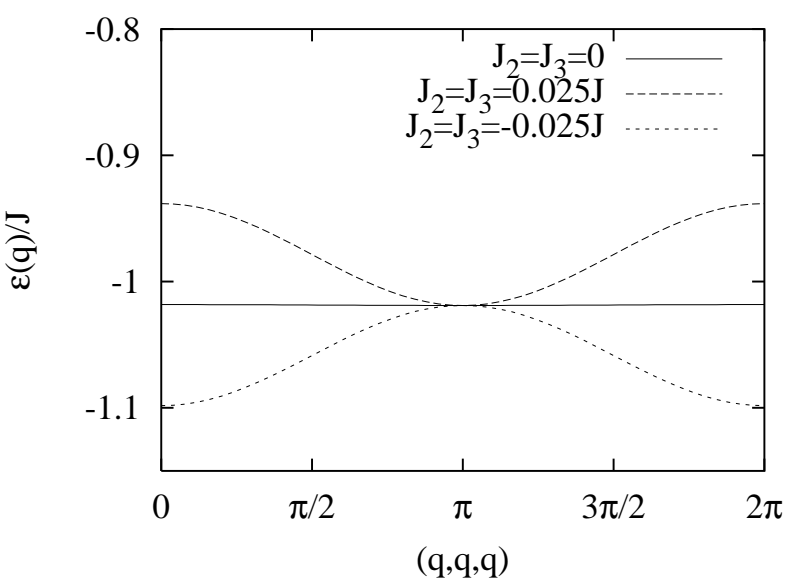

FIG. 4: The lowest part of the spectrum of $J(\mathbf{q})$ (from Ref. [10]). One sees that the degeneracy of the critical soft modes is lifted by second and third neighbor couplings. A positive (resp. negative) $J_{2}=J_{3}$ favors $\mathbf{q}=\boldsymbol{\pi}$ (resp. $\mathbf{q}=0$ ).

higher temperatures, see Figs. 5 and 6 . This is expected from Fig. 4 since, with $J_{2}=J_{3}<0, J(\mathbf{q})$ acquires a well-defined minimum at $\mathbf{q}=0$ which gets deeper with increasing $J_{2}$ and $J_{3}$.

Even with couplings as small as $J_{2}=J_{3}=-0.061 \mathrm{~J}$, the order parameter and the Binder ratio, shown in Fig. 6 , vary in a much more gradual way than for the degenerate case $J_{2}=J_{3}=0$. Although only finite-size scaling on a bigger range of sizes could say whether the transition is first or second order, it is clear that removing the degeneracy makes the transition less first-order compared with the degenerate case. These results are consistent with earlier simulations on model without dipole-dipole interactions (where larger clusters could be considered) which pointed out a continuous ${ }^{25}$ or weakly first-order ${ }^{26}$ transition for the pyrochlore lattice with $J_{3}<0$.

Next we consider $J_{2}=J_{3}>0$ which, from Fig. 4 , is expected to favor the B-like states $(\mathbf{q}=\boldsymbol{\pi})$, and indeed this is the case as shown by Figs. 7 and 8. The minimum of $J(\mathbf{q})$ at $\mathbf{q}=\boldsymbol{\pi}$ shown in Fig. 4 is independent of $J_{2}$ and $J_{3}$ which implies that the mean-field transition temperature is also independent of $J_{2}$ and $J_{3}$. Although we do not have enough system sizes to attempt a serious estimate of $T_{N}$ using finite size scaling, it seems that the transition temperature is indeed quite similar to that for $J_{2}=J_{3}=0$.

As was also found for $J_{2}=J_{3}<0$, the transition is much more gradual than for the degenerate case, showing that removing the degeneracy reduces the first-order character of the transition. The sensitive dependence of both the order of the transition and the nature of the ordered phase on $J_{2}$ and $J_{3}$ points to the possible relevance of these terms in explaining the difference between $\mathrm{Gd}_{2} \mathrm{Sn}_{2} \mathrm{O}_{7}$ and $\mathrm{Gd}_{2} \mathrm{Ti}_{2} \mathrm{O}_{7}$.

While the data for $J_{2}=J_{3}=0$ clearly indicate a strong first order transition, our results for $J_{2}$ and $J_{3}$ nonzero (with either sign) are not conclusive as to the order 


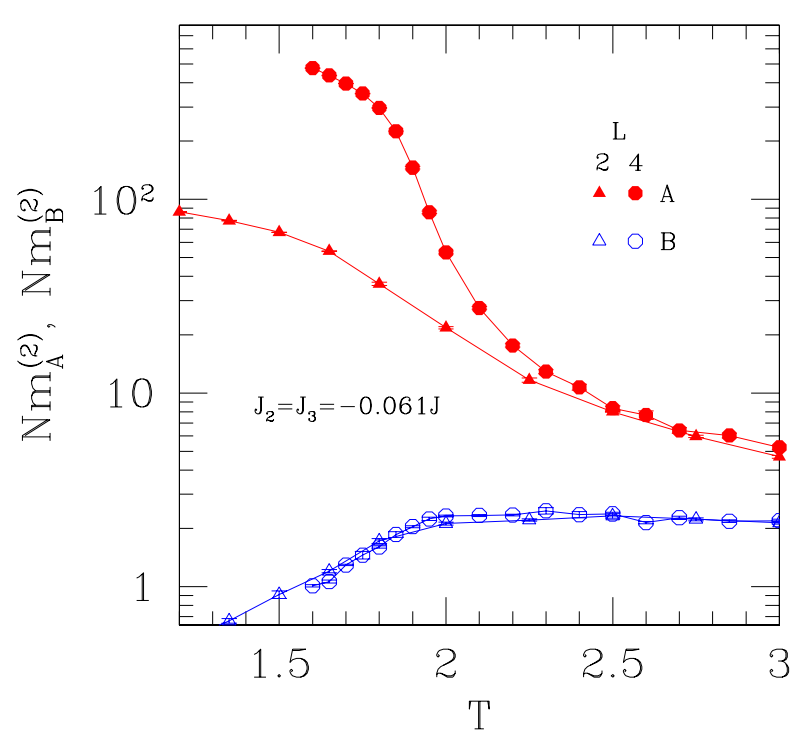

FIG. 5: (Color online). Order parameters squared $(\times N)$ for $J_{2}=J_{3}=-0.061 J$. A transition occurs to the A phase $(\mathbf{q}=0)$ which is more gradual than for the degenerate case with $J_{2}=J_{3}=0$ shown in Fig. 2.

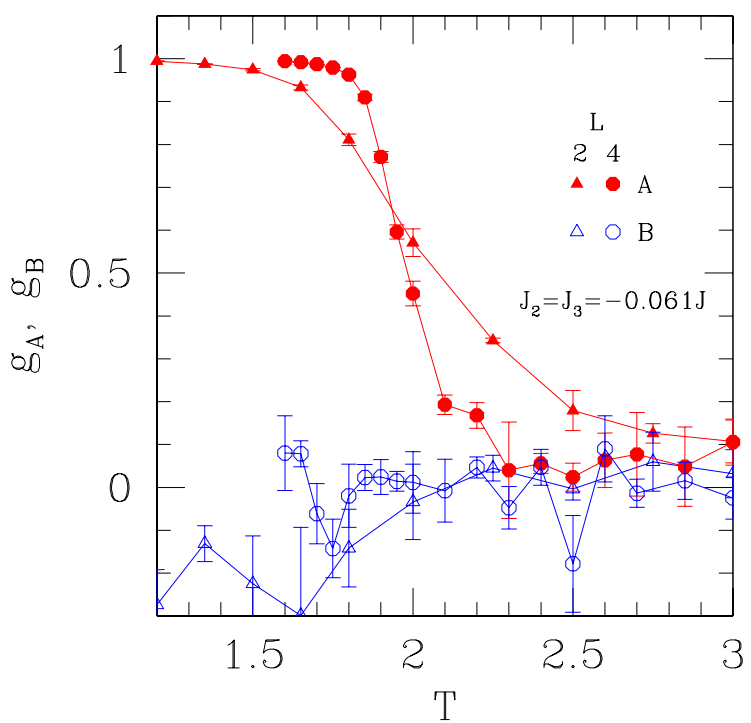

FIG. 6: (Color online). The Binder ratio for the $\mathrm{A}$ and $\mathrm{B}$ phases for different system sizes $L$ for $J_{2}=J_{3}=-0.061 J$.

of the transition. The smooth behavior of the Binder ratios shown in Figs. 6 and 8 is typical for a second order transition. However, we cannot rule out the possibility of a weak first order transition where the correlation length at the critical point, $\xi_{c}$, is large. In this case, if $L<\xi_{c}$ the behavior will look like that of a second order transition. Only for sizes where $L>\xi_{c}$ can one see a crossover to behavior expected at a first order transition. Hence for $J_{2}$ and $J_{3}$ non-zero, where the degeneracy is removed, the transition is either second order or weakly first order. However, comparing Figs. 6 and 8 with the corresponding figure for $J_{2}=J_{3}=0$, Fig. 3 , we see that behavior for $J_{2}$ and $J_{3}$ non-zero is very different from the strong first order behavior found in the degenerate case.

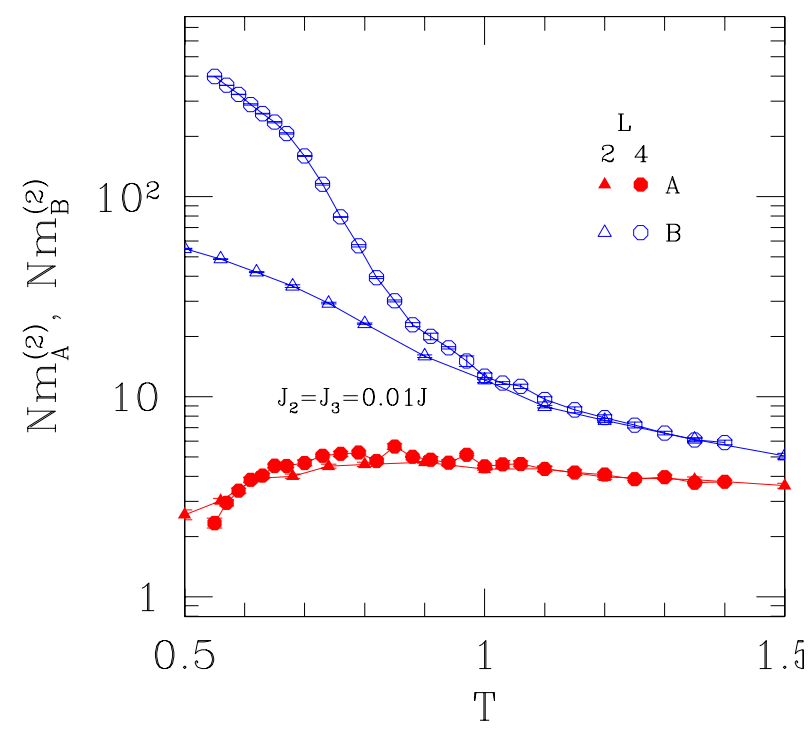

FIG. 7: (Color online). Order parameters obtained by Monte Carlo simulation for $J_{2}=J_{3}=0.01 J$. The transition gives rise to the B-like state $(\mathbf{q}=\boldsymbol{\pi})$ and is more gradual than for the case of $J_{2}=J_{3}=0$ shown in Fig. 2.

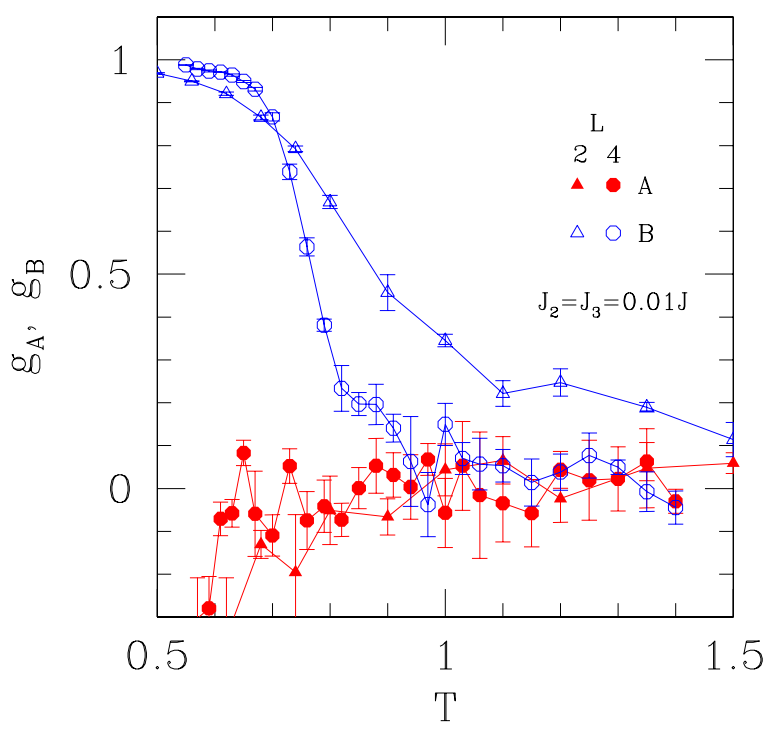

FIG. 8: (Color online). Binder ratio for the $\mathrm{A}$ and $\mathrm{B}$ phases for different system sizes $L$ for $J_{2}=J_{3}=0.01 \mathrm{~J}$.

\section{RG ANALYSIS OF THE $(1,1,1)$ MODEL}

We now study a Landau-Ginzburg-Wilson model by means of the renormalization group analysis. Although the method is usually aimed to study second-order phase transitions, the lack of stable fixed points is often considered as an indication for a first-order kind of transition.

Given the degeneracies of the soft modes with $\mathbf{q}$ along the 4 equivalent $(1,1,1)$ directions when $J_{2}=J_{3}=0$ (see 
Fig. 4), the fluctuations of all these modes must be taken into account simultaneously. For this reason, we consider a model with an infinite-component order parameter (extended to dimension $d$ ) and the fluctuations with wavevectors close to these soft mode directions. The quadratic part of the Hamiltonian is written:

$$
\begin{aligned}
H^{(2)} & =\int \frac{d^{d} \mathbf{q}}{(2 \pi)^{d}} \sum_{i=1}^{4} \mathcal{G}_{0 i}^{-1}(\mathbf{q}) \psi_{i \mathbf{q}} \bar{\psi}_{i \mathbf{q}} \\
\mathcal{G}_{0 i}^{-1}(\mathbf{q}) & =\left(r+\mathbf{q}_{\perp, i}^{2}+a q_{\|, i}^{2 m}\right) \\
\mathbf{q}_{\perp, i} & =\mathbf{q}-\left(\hat{v}_{i} \cdot \hat{\mathbf{q}}\right) \mathbf{q}
\end{aligned}
$$

where the $\hat{v}_{i}$ are of norm 1 and represent the $i=1, \ldots, 4$ $(1,1,1)$ directions given in Fig. 9. We have 8 fields, $\psi_{i \mathbf{q}}$ and $\bar{\psi}_{i \mathbf{q}}(i=1, \ldots, 4)$, with $\bar{\psi}_{i \mathbf{q}}=\psi_{-i,-\mathbf{q}}=\psi_{i \mathbf{q}}^{*}$. If we ignore the $a q_{\|, i}^{2 m}$ term, then, when $r=0$, all the modes with $\mathbf{q}_{\perp, i}=0$ become simultaneously unstable. However, as in previous works,${ }^{20,21}$ we include the small dispersion along the $(1,1,1)$ lines, $a q_{\|, i}^{2 m}$, to make the calculation well defined at intermediate stages. To study the degenerate case, it will be eliminated at the end of the calculation by taking $m \rightarrow+\infty$.

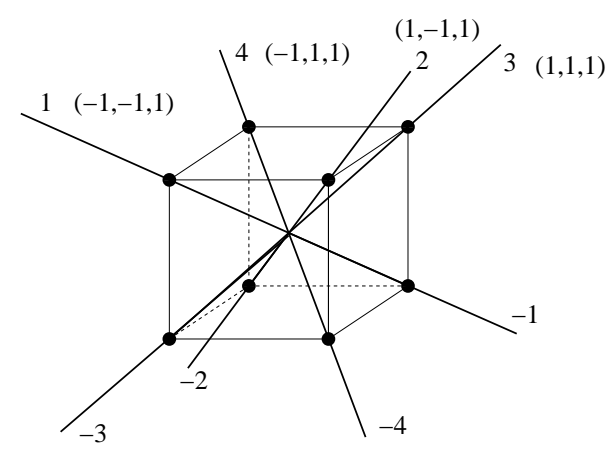

FIG. 9: Lines of minimum energy in reciprocal space, given by the 4 equivalent $(1,1,1)$ directions (the cube is drawn for convenience). The (lack of) dispersion along the $(1,1,1)$ lines is shown in Fig. 4.

The fourth-order invariants are similar to that introduced to describe the nematic smectic- $\mathrm{C}$ transitions ${ }^{20}$ and are given by:

$$
H^{(4)}=\int \frac{d^{d} \mathbf{q}_{1}}{(2 \pi)^{d}} \frac{d^{d} \mathbf{q}_{2}}{(2 \pi)^{d}} \frac{d^{d} \mathbf{q}_{3}}{(2 \pi)^{d}} \frac{d^{d} \mathbf{q}_{4}}{(2 \pi)^{d}} \delta_{\mathbf{q}_{1}, \mathbf{q}_{2}, \mathbf{q}_{3}, \mathbf{q}_{4}} \mathcal{H}_{4}
$$

where $\delta_{\mathbf{q}_{1}, \mathbf{q}_{2}, \mathbf{q}_{3}, \mathbf{q}_{4}}$ ensures that the total momentum of the four $\psi$ is zero. For instance, we can cancel the momentum by choosing pairs of momenta along the same $(1,1,1)$ line, i.e. by combining $\psi_{i, \mathbf{q}_{1}}$ with $\psi_{-i, \mathbf{q}_{2}=-\mathbf{q}_{1}}$, and similarly with $\mathbf{q}_{3}$ and $\mathbf{q}_{4}$. That gives a fourth-order term $u_{p} \psi_{i} \bar{\psi}_{i} \psi_{i+p} \bar{\psi}_{i+p}$ term, where $i=1, \ldots, 4$ and $(i+p)$ is meant for $(i+p-4)$ if $(i+p)>4$. Note that given the $C_{4}$ symmetry, $u_{p}$ does not depend upon $i$, but only upon $p=0,1,2$. In addition, we could choose the first two q's along 1 and 3 for instance (see Fig. 9) and the other two along -2 and -4 , which gives $\psi_{1} \bar{\psi}_{2} \psi_{3} \bar{\psi}_{4}$. Another simplifying feature that we have adopted consists of neglecting the wave-vector dependence of the coefficients $u_{p}$. Omitting to write the $\mathbf{q}_{\mathbf{1}, \mathbf{2 , 3}, \mathbf{4}}$ wave-vectors, the only fourth-order invariants are given by:

$$
\begin{aligned}
\mathcal{H}_{4} & =\sum_{p=0}^{2} u_{p} \sum_{i=1}^{4} \psi_{i} \bar{\psi}_{i} \psi_{i+p} \bar{\psi}_{i+p} \\
& +\frac{1}{2} u_{3}\left(\psi_{1} \bar{\psi}_{2} \psi_{3} \bar{\psi}_{4}+\bar{\psi}_{1} \psi_{2} \bar{\psi}_{3} \psi_{4}\right)
\end{aligned}
$$

where $\bar{\psi}_{i}=\psi_{-i}$. We call $H^{(2)}+H^{(4)}$ the $(1,1,1)$ model. First, we consider the Hartree correction ${ }^{19}$ to the gap $r$ (self-energy):

$$
\begin{aligned}
\mathcal{G}_{i}^{-1} & \equiv r+\mathbf{q}_{\perp i}^{2}+\Sigma_{i}(r) \\
\Sigma_{i}(r) & =\frac{1}{6}\left(3 u_{0}+2 u_{1}+u_{2}\right) \int \frac{d^{d} \mathbf{q}}{(2 \pi)^{d}} \mathcal{G}_{0 i}(\mathbf{q})
\end{aligned}
$$

In $d=3$, if we introduce momentum cut-offs, the new gap $r^{\prime}(a \rightarrow 0)$ is given by:

$$
r^{\prime}=r+\alpha \Lambda^{\prime} \ln \left(1+\frac{\Lambda^{2}}{r^{\prime}}\right)
$$

where $\alpha$ is a proportionality coefficient and $\Lambda$ and $\Lambda^{\prime}$ the cut-offs. Due to the strong singularity of the right-handside, the gap does not vanish anymore. It suggests that the paramagnetic phase remains locally stable below the transition, together with other more stable phases. From the existence of other phases (at least at the mean-field level), the transition is expected to be first-order. ${ }^{19}$

However, the cut-offs $\Lambda$ and $\Lambda^{\prime}$ enter explicitly the equation and a more controlled result can be obtained by the renormalization group analysis by restricting the integration to a shell of momentum $\Lambda / b<q<\Lambda$ with $b>1 .^{20,21}$ For this we introduce new real orderparameters $(i=1, \ldots, 4)$ :

$$
\psi_{i}=\phi_{i}+i \bar{\phi}_{i} \quad \bar{\psi}_{i}=\phi_{i}-i \bar{\phi}_{i}
$$

and the quadratic and quartic terms become:

$$
\begin{aligned}
\mathcal{H} & =\sum_{i=1}^{4} \mathcal{G}_{0 i}^{-1}\left(\phi_{i}^{2}+\bar{\phi}_{i}^{2}\right)+u_{0} \sum_{i=1}^{4}\left(\phi_{i}^{2}+\bar{\phi}_{i}^{2}\right)^{2} \\
& +u_{1}\left[\left(\phi_{1}^{2}+\bar{\phi}_{1}^{2}\right)+\left(\phi_{3}^{2}+\bar{\phi}_{3}^{2}\right)\right]\left[\left(\phi_{2}^{2}+\bar{\phi}_{2}^{2}\right)+\left(\phi_{4}^{2}+\bar{\phi}_{4}^{2}\right)\right] \\
& +u_{2}\left[\left(\phi_{1}^{2}+\bar{\phi}_{1}^{2}\right)\left(\phi_{3}^{2}+\bar{\phi}_{3}^{2}\right)+\left(\phi_{2}^{2}+\bar{\phi}_{2}^{2}\right)\left(\phi_{4}^{2}+\bar{\phi}_{4}^{2}\right)\right] \\
& +u_{3}\left(\phi_{1} \phi_{2} \phi_{3} \phi_{4}+\bar{\phi}_{1} \bar{\phi}_{2} \bar{\phi}_{3} \bar{\phi}_{4}\right) \\
& -u_{3}\left(\phi_{1} \bar{\phi}_{2} \phi_{3} \bar{\phi}_{4}+\bar{\phi}_{1} \phi_{2} \bar{\phi}_{3} \phi_{4}\right) \\
& +u_{3}\left(\phi_{1} \bar{\phi}_{3}+\bar{\phi}_{1} \phi_{3}\right)\left(\phi_{2} \bar{\phi}_{4}+\bar{\phi}_{2} \phi_{4}\right)
\end{aligned}
$$

The derivation of the RG equations for the coupling constants is then similar to that of Ref. [20] except that we have to keep track of the field labels, given the anisotropy of $\mathcal{G}_{0 i}$ in Eq. (15). By integrating over a shell of momentum $\Lambda / b<q<\Lambda$, we find the recursion relations for the 
new coupling constants:

$$
\begin{aligned}
u_{0}^{\prime} & =b^{\epsilon}\left[u_{0}-\left(40 u_{0}^{2} I_{0}+4 u_{1}^{2} I_{0}+2 u_{2}^{2} I_{0}\right)\right] \\
u_{1}^{\prime} & =b^{\epsilon}\left[u_{1}-\left(8 u_{1}^{2} I_{1}+u_{3}^{2} I_{1}+32 u_{0} u_{1} I_{0}+8 u_{1} u_{2} I_{0}\right)\right] \\
u_{2}^{\prime} & =b^{\epsilon}\left[u_{2}-\left(8 u_{1}^{2} I_{0}+8 u_{2}^{2} I_{2}+u_{3}^{2} I_{2}+32 u_{0} u_{2} I_{0}\right)\right] \\
u_{3}^{\prime} & =b^{\epsilon}\left[u_{3}-\left(8 u_{2} u_{3} I_{2}+8 u_{1} u_{3} I_{1}\right)\right] \\
a^{\prime} & =b^{-2(m-1)} a
\end{aligned}
$$

where $\epsilon=4-d$ and the integrals are defined by

$$
I_{p}=\int_{\Lambda / b}^{\Lambda} \frac{d^{d} \mathbf{q}}{(2 \pi)^{d}} \mathcal{G}_{0 i}(\mathbf{q}) \mathcal{G}_{0 i+p}(\mathbf{q})
$$

\section{A. Degenerate $(\mathbf{1}, \mathbf{1}, \mathbf{1})$ model $(a \rightarrow 0)$}

The relation (16), together with the divergences of the integrals $I_{p}$ for $a \rightarrow 0$ (at $r=0$, finite $b$ ) implies that $a$ is a dangerous irrelevant variable for $m>1$. $I_{0}$ diverges indeed as $a^{(d-5) / 2}$, and $I_{1,2}$ as $a^{(d-3) / 2}$. To take into account these divergences, we have to introduce rescaled constants $\tilde{u}_{i}=u_{i} a^{(d-5) / 2}$ (Ref. $[20,21]$ and references therein). With $a^{(5-d) / 2} I_{1,2} \rightarrow 0$, the recursion relations become (we introduce $\mathcal{I} \equiv \lim _{a \rightarrow 0} a^{(5-d) / 2} I_{0}$ ):

$$
\begin{aligned}
& \tilde{u}_{0}^{\prime}=b^{m(5-d-1 / m)}\left[\tilde{u}_{0}-\left(40 \tilde{u}_{0}^{2}+4 \tilde{u}_{1}^{2}+2 \tilde{u}_{2}^{2}\right) \mathcal{I}\right] \\
& \tilde{u}_{1}^{\prime}=b^{m(5-d-1 / m)}\left[\tilde{u}_{1}-\left(32 \tilde{u}_{0} \tilde{u}_{1}+8 \tilde{u}_{1} \tilde{u}_{2}\right) \mathcal{I}\right] \\
& \tilde{u}_{2}^{\prime}=b^{m(5-d-1 / m)}\left[\tilde{u}_{2}-\left(8 \tilde{u}_{1}^{2}+32 \tilde{u}_{0} \tilde{u}_{2}\right) \mathcal{I}\right] \\
& \tilde{u}_{3}^{\prime}=b^{m(5-d-1 / m)} \tilde{u}_{3}
\end{aligned}
$$

All the fixed points are unstable for $d<5-1 / m$ (the upper critical dimension is 5 for $m \rightarrow \infty$ ) since $\tilde{u}_{3}^{\prime}=b^{m(5-d-1 / m)} \tilde{u}_{3}$. Although strictly speaking new fixed points could occur at order $\epsilon^{2}$, the present calculation at order $\epsilon$ is compatible with the first-order transition observed in the Monte-Carlo simulations.

\section{B. No Degeneracy}

We now remove the degeneracy, e.g. by including further neighbor couplings in the microscopic Hamiltonian.

$$
\text { 1. } \mathbf{q}=\mathbf{0}
$$

First of all we assume that the ordering is at $\mathbf{q}=0$. It is simplest to go back to the order parameter shown in Fig. 1, realize that there are three components $\psi_{i}, i=$ $1,2,3$, and that the symmetry is cubic. The Hamiltonian of this cubic model is therefore given by

$$
\mathcal{H}=\sum_{i=1}^{3}\left[\left(r+\mathbf{q}^{2}\right) \psi_{i}^{2}+u_{0} \psi_{i}^{4}\right]+u_{1} \sum_{i, j} \psi_{i}^{2} \psi_{j}^{2}
$$

There has been a controversy regarding whether the stable fixed point of the cubic model is the Heisenberg or the cubic fixed point. A recent 6-loop expansion has shown that for $n>2.89$, the stable fixed-point is the cubic one. ${ }^{27}$ Depending on the initial values for the coupling constants, the transition could be either first-order or continuous. However, to stabilize the collinear states (with $\psi$ either $(1,0,0),(0,1,0)$, or $(0,0,1)$ and $\mathbf{q}=0)$, the set of initial coupling constants leads to a first-order transition. ${ }^{28}$

We have seen that a first order transition is obtained both in the degenerate case and also when there is a well-defined minimum at $\mathbf{q}=0$. Is, then, the degeneracy of soft modes important or not? We note that, in the absence of degeneracy, the transition may be only weakly first-order. The problem was studied some years ago in the context of the pyrochlore $\mathrm{FeF}_{3}$. For this compound, the $\mathbf{q}=0$ state found by neutron scattering was characterized by a 3-component order parameter, ${ }^{25}$ similar to the one we have here. Monte-Carlo simulations have shown that the collinear structures with $\psi=(1,0,0) ;(0,1,0) ;(0,0,1)$ are preferred, but the transition first appeared to be second-order, with unusual critical exponents ${ }^{25}$ contrary to the $\mathrm{RG}$ argument given above. We can reconcile these results by suggesting that the transition may be weakly first-order, so that the correlation length would exceed the size of the Monte-Carlo cluster and the transition would appear second-order in the simulation. This is also comforted by a reexamination of the Monte-Carlo results, which suggested that the transition is more likely to be indeed weakly first-order. ${ }^{26}$

$$
\text { 2. } \mathbf{q}=\boldsymbol{\pi}
$$

We now assume that the degeneracy is lifted in such a way that one of the four $\boldsymbol{\pi}$ wave-vectors is selected. Since $\boldsymbol{\pi}$ and $-\boldsymbol{\pi}$ are related by a reciprocal lattice vector, we have to take into account the fluctuations of four fields only, with $\mathbf{q}$ close to any of the $\boldsymbol{\pi}$ wave-vectors, $\psi_{i}, i=$ $1, \ldots, 4$. The critical model is given by

$$
\begin{aligned}
\mathcal{H} & =\sum_{i=1}^{4}\left[\left(r+\mathbf{q}^{2}\right) \psi_{i}^{2}+u_{0} \psi_{i}^{4}\right]+u_{1} \sum_{i, j} \psi_{i}^{2} \psi_{j}^{2} \\
& +u_{3} \psi_{1} \psi_{2} \psi_{3} \psi_{4} .
\end{aligned}
$$

This model is known to possess unstable fixed points at order $\epsilon^{2} .^{18}$ Therefore the transition to the $\boldsymbol{\pi}$ phases is also expected to be first-order.

On the basis of the LGW models alone, we would conclude that the phase transitions in the dipolar pyrochlore are all first-order in character. Such a simple analysis does not say whether the transition is strongly or weakly first-order that is quite a relevant question when one comes to compare with experiments. Nonetheless, the results presented in this section are compatible with the Monte-Carlo simulations of section II. The latter are important, precisely to say whether the transitions are weakly or strongly first-order. 


\section{CONCLUSION}

We have considered the dipolar Heisenberg model on a pyrochlore lattice with nearest neighbor interactions and a small amount of second and third neighbor interactions $\left(J_{2}\right.$ and $\left.J_{3}\right)$. For $J_{2}=J_{3}=0$ the system is highly degenerate, see Fig. 4, and fluctuation effects pick out ordering at $\mathbf{q}=0$ (A-type). Monte Carlo simulations show that the transition is very strongly first order in this case, in contrast to mean-field theory which predicts a second order transition. A first order transition is also predicted by a renormalization group analysis. When the degeneracy is removed by including $J_{2}$ and $J_{3}$ the transition is more gradual, showing that the degeneracy is necessary to get a strong first order transition. Given the limited range of sizes in the Monte Carlo simulations, we cannot say from the simulations whether the transition is second order or weakly first order for $J_{2}=J_{3} \neq 0$. However, according to a renormalization group analysis for the non-degenerate case, both A and B type orderings have no stable fixed points, indicating, presumably, a fluctuation induced first order transition. Usually this type of transition is only weakly first order, and this seems to be consistent with our numerical data.

Because of the degeneracy for $J_{2}=J_{3}=0$, a small amount of second and third neighbor coupling can also change the nature of the ground state. We find that for $J_{2}=J_{3}<0$ the A phase is retained but for $J_{2}=J_{3}>0$ we obtain a $\mathbf{q}=\boldsymbol{\pi}$ (B-type) ordering. In future work we will study in more detail the nature of this B-type phase, and also consider other possible phases that occur when $J_{2} \neq J_{3}$. It is possible that anisotropic interactions, suggested on the basis of high-temperature $\mathrm{ESR}^{29}$ and by EPR on diluted samples, ${ }^{30,31}$ may be needed to explain the experimentally observed phases in detail.
Our results provide a natural explanation for $\mathrm{Gd}_{2} \mathrm{Sn}_{2} \mathrm{O}_{7}$ having a strong first-order transition, ${ }^{4}$ while $\mathrm{Gd}_{2} \mathrm{Ti}_{2} \mathrm{O}_{7}$ has a second-order transition ${ }^{12}$ (though a weak first-order transition is not ruled out experimentally); namely second and third neighbor interactions are very weak in $\mathrm{Gd}_{2} \mathrm{Sn}_{2} \mathrm{O}_{7}$, but they are stronger and positive for $\mathrm{Gd}_{2} \mathrm{Ti}_{2} \mathrm{O}_{7}$. In this respect, ab-initio calculations could give some estimate of the strength of the couplings. This picture is also consistent with the observations that $\mathrm{Gd}_{2} \mathrm{Ti}_{2} \mathrm{O}_{7}$ orders ${ }^{12,13}$ at $\mathbf{q}=\boldsymbol{\pi}$ while $\mathrm{Gd}_{2} \mathrm{Sn}_{2} \mathrm{O}_{7}$ should be A-type with equivalent sites and moments perpendicular to the local $(1,1,1)$ directions. ${ }^{14}$

In the presence of a magnetic-field, ${ }^{5,10} \mathrm{Gd}_{2} \mathrm{Ti}_{2} \mathrm{O}_{7}$ has a rich phase diagram. For the future, it would also be interesting to perform a study of field-induced transitions in $\mathrm{Gd}_{2} \mathrm{Sn}_{2} \mathrm{O}_{7}$, since this starts off with a quite different state in zero field. In addition to the multiple phase transitions expected on the basis of mean-field theory, the field reduces the fluctuations and, hence should reduce the strong first-order character of the transition.

\section{Acknowledgments}

We would like to thank A. Wills for sharing with us unpublished results concerning $\mathrm{Gd}_{2} \mathrm{Sn}_{2} \mathrm{O}_{7}$. O.C. would like to thank especially G. Jackeli and T. Ziman for very stimulating discussions and also S. Bramwell, V. Glazkov, E. Kats, L. Lévy, Y. Motome, R. Stewart, and M. Zhitomirsky. A.P.Y. acknowledges support from the National Science Foundation under Grant No. DMR 0337049. B.S.S. acknowledges support from the National Science Foundation under Grant No. DMR 0408247. A.P.Y. would also like to thank the Institut Laue Langevin, where this work was started, for its hospitality.
1 J. N. Reimers, Phys. Rev. B 45, 7287 (1992).

2 R. Moessner and J. T. Chalker, Phys. Rev. Lett. 80, 2929 (1998); Phys. Rev. B 58, 12049 (1998).

3 N. P. Raju, M. Dion, M. J. P. Gingras, T. E. Mason, J. E. Greedan, Phys. Rev. B 59, 14489 (1999).

4 P. Bonville, J. A. Hodges, M. Ocio, J.-P. Sanchez, P. Vulliet, S. Sosin and D. Braithwaite, J. Phys. Condens. Matter 15, 7777 (2003).

5 A. P. Ramirez, B. S. Shastry, A. Hayashi, J. Krajewski, D. Huse and R. J. Cava, Phys. Rev. Lett. 89, 067202 (2002).

${ }^{6}$ O. A. Petrenko, M. R. Lees, G. Balakrishnan, and D. McK Paul, Phys. Rev. B 70, 012402 (2004).

7 Strictly speaking the degeneracy is only approximate. It is lifted in favor of $\mathbf{q}=(\pi, \pi, \pi)$ by a very small amount. ${ }^{9-11}$

8 S. E. Palmer and J. T. Chalker, Phys. Rev. B 62, 488 (2000).

9 D. A. Huse (unpublished).

10 O. Cépas and B. S. Shastry, Phys. Rev. B 69, 184402 (2004).

11 M. Enjalran, and M.J.P. Gingras, cond-mat/0307152 (unpublished).
12 J. D. M. Champion, A. S. Wills, T. Fennell, S. T. Bramwell, J. S. Gardner and M. A. Green, Phys. Rev. B 64, 140407 (2001).

13 J. R. Stewart, G. Ehlers, A. S. Wills, S. T. Bramwell, and J. S. Gardner, J. Phys. Condens. Matter 16, L321 (2004).

14 E. Bertin, J. A. Hodges, J.-P. Bouchaud, P. Bonville, J.-P. Sanchez and P. Vuillet, Eur. Phys. J. B 27, 347 (2002).

15 E. Brézin, J. C. Le Guillou, and J. Zinn-Justin, Phys. Rev. B 10, 892 (1974).

16 P. Bak, S. Krinsky, and D. Mukamel, Phys. Rev. Lett. 36, 52 (1976).

17 D. Mukamel and S. Krinsky, Phys. Rev. B 13, 5065 (1976).

18 D. Mukamel and S. Krinsky, ibid. 13, 5078 (1976).

19 S. A. Brazovskii, Sov. Phys.-JETP, Vol. 41, 85 (1975) [Zh. Eksp. Teor. Fiz. 68, 175 (1975)].

20 D. Mukamel, and R. M. Hornreich, J. Phys. C: Solid St. Phys. 13, 161 (1980).

21 D. D. Ling, B. Friman, G. Grinstein, Phys. Rev. B 24, 2718 (1981).

22 K. Hukushima, and K. Nemoto, J. Phys. Soc. Jpn. 65, 1604 (1996); E. Marinari in Advances in Computer Simulation 
(Editors J. Kertész and I. Kondor, Springer-Verlag, Berlin, 1998) p. 50 (also in cond-mat/9612010).

23 O. A. Petrenko, and D. McK. Paul, Phys. Rev. B 63, 024409 (2000).

${ }^{24}$ K. Binder, and D. P. Landau, Phys. Rev. B 30, 1477 (1984).

25 J. N. Reimers, J. E. Greedan, and M. Björgvinsson, Phys. Rev. B 45, 7295 (1992).

26 A. Mailhot, and M. L. Plumer, Phys. Rev. B 48, 9881 (1993).

27 J. M. Carmona, A. Pelissetto, and E. Vicari, Phys. Rev. B 61,15136 (2000).
28 D. J. Amit, Field Theory, the Renormalization Group, and Critical Phenomena, p.330-336, Revised Second Edition, World Scientific, 1978.

29 A. K. Hassan, L. P. Lévy, C. Darie and P. Strobel, Phys. Rev. B 67, 214432 (2003).

${ }^{30}$ V. N. Glazkov, M. E. Zhitomirsky, A. I. Smirnov, H.-A. Krug von Nidda, A. Loidl, C. Marin, and J.-P. Sanchez, Phys. Rev. B 72, 020409(R) (2005).

31 We have checked that a sizeable on-site easy-plane anisotropy as found in ref. [30] does not change the degeneracy of the soft modes. 